\title{
Knochenbruchheilung und klinische Belastungsstabilität
}

Gabriele Rußow, Mark Heyland, Sven Märdian, Georg N. Duda

\section{Historie}

Die Vorteile der Stabilisierung von Frakturen zur Knochenbruchheilung sind bereits seit Jahrtausenden bekannt. Erste Schriften, die anstelle der einfachen Schienung mit Holzstäben eine Gipstherapie zur Stabilisierung empfahlen, stammen aus dem 10. Jahrhundert nach Christus in Persien [2]. Eine Adaptation solcher Konzepte in den westlichen Ländern folgte erst nach Ende des Mittelalters, indem die Extremität in einen Kasten aus Holz gelegt und mit Gips ausgefüllt wurde. Die so erstellten Stabilisierungen waren umständlich, schwer und mit einer Immobilisierung des Patienten bis zur Frakturkonsolidierung verbunden. Zusätzlich waren diese Strategien mit einem hohen Risiko an Komplikationen wie Thrombosen und Pneumonien, nach Konsolidierung mit Gelenkkontrakturen und Muskelatrophie verbunden. Erst 1851 konnten durch den Begründer des modernen Gipsverbandes, dem Niederländer Antonius Mathijsen [3], grundsätzlich neue Wege beschritten werden: Sein Konzept der Stabilität beinhaltete einen Gips, der aus der Kombination einer Stoffbandage und dem Gipspulver die Fragmentstabilisierung als äußere Schienung ermöglichte und der betroffenen Extremität anmodelliert wurde.

Schon vor Einführung des Gipsverbandes sind erste Ansätze zur internen Stabilisierung mithilfe einer Osteosynthese von Azteken und Inkas in Mittelamerika durchgeführt worden. Die Konquistadoren beschrieben die Therapie von Pseudarthrosen langer Röhrenknochen mittels intramedullärer Schienung durch Holzstäbe bereits im 16. Jahrhundert [4]. Ab dem 19. Jahrhundert wurden auch in Europa verschiedenste Verfahren zur operativen Stabilisierung von Frakturen beschrieben und fanden nach Einführung der antiseptischen Operationslehre durch Joseph Lister auch zunehmend Verbreitung. Der Begriff Osteosynthese wurde vom belgischen Chirurgen Albin Lambotte geprägt, der die Verfahrensentwicklung sowohl der Osteosynthese mittels Fixateur externe als auch der Plattenosteosynthese erheblich beeinflusste [5].

Obwohl der von Lambottes Landsmann und Kollegen Robert Danis geprägte Satz, der Frakturkallus sei „eine pathologische Struktur und seine Bildung müsse mit Osteosynthesen verhindert werden“, nicht mehr uneingeschränkt gilt, so hat dessen Werk „Theorie und Praxis der Osteosynthese“ (1949) maßgeblich zur Begründung der
Arbeitsgemeinschaft für Osteosynthesefragen (AO) durch die Schweizer Chirurgen Maurice E. Müller, Robert Schneider, Hans Willenegger, und Martin Allgöwer beigetragen [6]. Diese Arbeitsgemeinschaft hat sich das Ziel gesetzt, Indikationen und Nutzen der operativen Frakturtherapie empirisch zu evaluieren und durch fundierte Wissenschaft eine Standardisierung der Therapiekonzepte zu erreichen. Seitdem sind wesentliche Konzepte für die Operationslehren, verfügbare Stabilisierungstechnologien, Instrumente und auch Implantate entstanden.

\section{Stabilität und Biologie}

Der umgangssprachlich oft verwendete Begriff „Stabilität" wird im Zusammenhang mit Osteosynthesen und Frakturheilung genutzt, um den mechanischen Begriff der Steifigkeit zu umschreiben. Die Steifigkeit bezeichnet den Widerstand eines Systems gegen elastische Verformung (Verschiebungen oder Verdrehungen) durch von außen einwirkende Lasten (Kräfte oder Momente) und wird in Kraft/Weg angegeben. Je größer die Steifigkeit einer Osteosynthese, desto weniger Verformung lässt sie unter Belastung an der Frakturzone zu. Eine geringe Verformung der Frakturzone ist somit durch entweder eine hohe Osteosynthesesteifigkeit oder eine geringe Belastung zu erreichen. Während die Belastung durch den Patienten gesteuert werden kann, ist die Steifigkeit der Osteosynthese durch den Operateur und dessen Techniken sowie das verwendete Material definiert. Um die erreichte Reposition zu retinieren und keine Instabilität an der Frakturzone zu erzeugen, ist eine stabile Osteosynthese essenziell. Dagegen ist für eine effektive Knochenheilung auch ein gewisser mechanischer Reiz (im Sinne von Mikrobewegungen) an der Frakturzone immanent, um eine schnelle und zügige Frakturkonsolidierung zu erreichen. Grundsätzlich sind für eine gute Knochenheilung neben der Wahl der Osteosynthese, den sich ergebenden lokalen mechanischen Bedingungen auch eine Reihe weiterer Aspekte wesentlich, die über den Heilungsverlauf entscheiden. Giannoudis et al. haben die lokal wirkenden Wachstumsfaktoren, das Vorhandensein osteogener also die Knochenheilung unterstützender - Zellen sowie die Angiogenese als die wesentlichen Faktoren der Knochenheilung definiert $[7,8]$. Heute wissen wir, dass zusätzlich noch der Wechsel von einem pro- zu einem antiinflammatorischen Milieu zentral für eine schnelle Heilung ist, und dass alle biologischen Parameter der Hei- 
lung (Angiogenese, Entzündung, Stammzellen, Zellmigration und Wachstumsfaktoren) in ihrer Effektivität und Effizienz mechanosensitiv sind. Neben den Weichteilverletzungen des Patienten bestimmen diese Parameter den Ablauf des Heilungsprozesses, können aber durch die mechanische Stabilisierung moduliert und gesteuert werden ( $\bullet$ Abb. 1) [1].

Zellen, insbesondere Osteoblasten, Osteozyten und Osteoklasten, sind mechanosensitiv: als Reaktion auf bestimmte mechanische Reize werden in den Zellen Signalkaskaden ausgelöst, die das Verhalten der Zellen und letztlich auch die Formation von neuem Knochen beeinflussen [9]. Bereits Ende des 19. Jahrhunderts wurde durch Julius Wolff nach Beobachtung der Trabekelstruktur in Femurköpfen und Schenkelhälsen nach Frakturen postuliert, dass sich Knochen entsprechend der mechanischen Belastung auf- und umbaut. Sein „Das Gesetz der Transformation der Knochen“ (1892) besagt unter anderem, dass Knochen bei vermehrter Belastung verstärkt wird und die Trabekelstruktur sich parallel zu den einwirkenden Kräften orientiert. Harold P. Frost postulierte in diesem Zusammenhang einen lebenslangen Umbau des gesamten Knochengerüstes entlang dieser mechanischen Einflüsse [10]. Er untersuchte postmortal und perioperativ entnommene Rippen von Patienten, die Tetrazykline nach abgeschlossener Skelettreifung eingenommen hatten, und konnte nachweisen, dass Tetrazykline unmittelbar unterhalb des Periosts in die Knochensubstanz durch die Osteoblastenumbauten eingelagert wurden. Frost schussfolgerte, dass es einen regulatorischen Zusammenhang zwischen mechanischen Reizen und knöchernen Umbauprozessen geben muss und entwickelte das sog. „Mechanostat“-Modell [11]. Angelehnt an technische Systeme zur Temperaturregelung postulierte Frost, dass bei Über-, bzw. Unterschreiten einer Belastungsgrenze Knochenmasse auf- bzw. abgebaut werden würde. Nach Frost lassen sich 4 Fenster für Knochenumbauprozesse definieren ( $\triangleright$ Abb. 2) [12]. Kommt es wiederholt zu Überlastungen, ohne dass zeitgleich auch ein passender knöcherner Umbau erfolgt, kann es zu Ermüdungsbrüchen kommen [13].

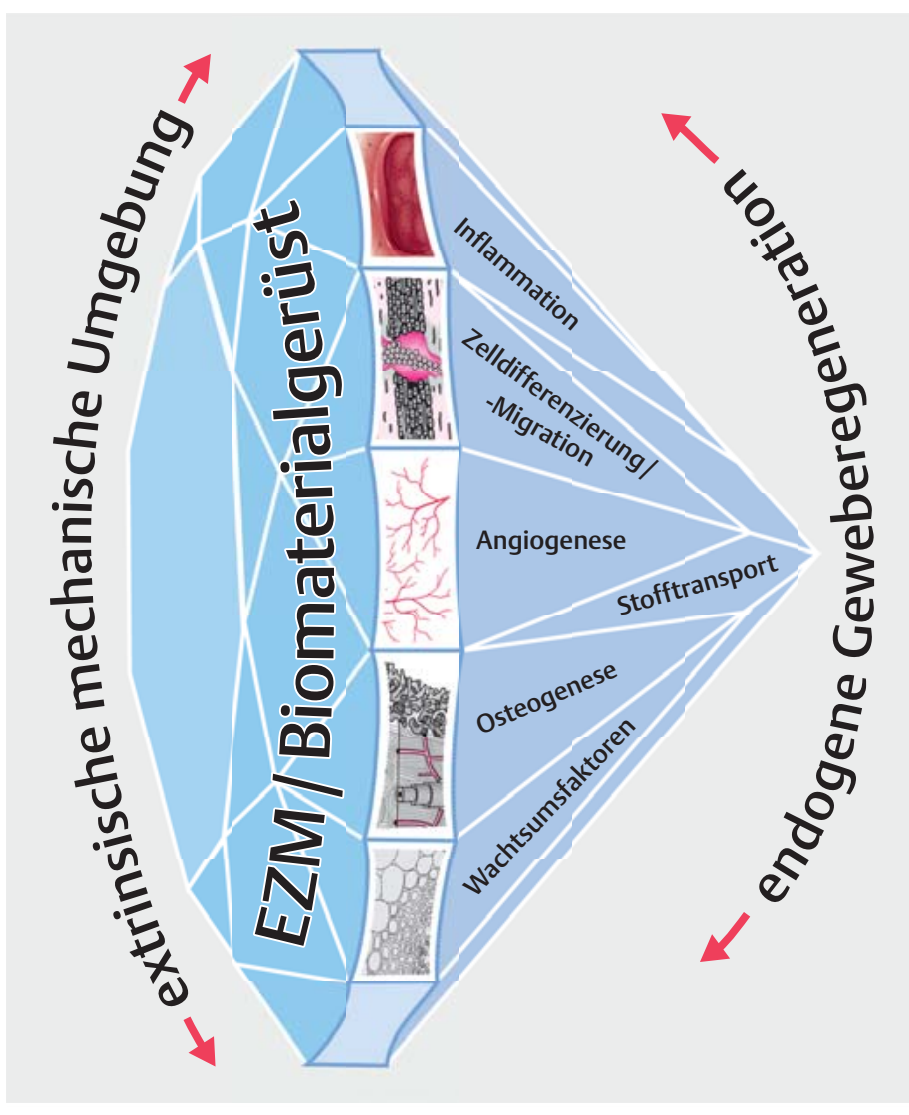

- Abb. 1 Diamond-Konzept der endogenen Knochenheilung.

Friedrich Pauwels postulierte Mitte des 20. Jahrhunderts, dass nicht nur reife Knochenzellen durch mechanische Kräfte beeinflusst werden, sondern bereits die Zelldifferenzierung von mesenchymalen Stammzellen in unterschiedliche Gewebsarten als Reaktion auf mechanische Reize erfolgt [14]. Bei hohen Zug- oder Scherkräften bildet sich aus mesenchymalen Stammzellen vorwiegend Bindegewebe aus. Bei deutlich reduzierten Scherkräften, jedoch noch mäßigen Zugkräften bildet sich vermehrt Knorpel aus. Nur bei geringen Zugkräften ohne wesentliche Scherkräfte kann eine ungestörte Knochenbildung

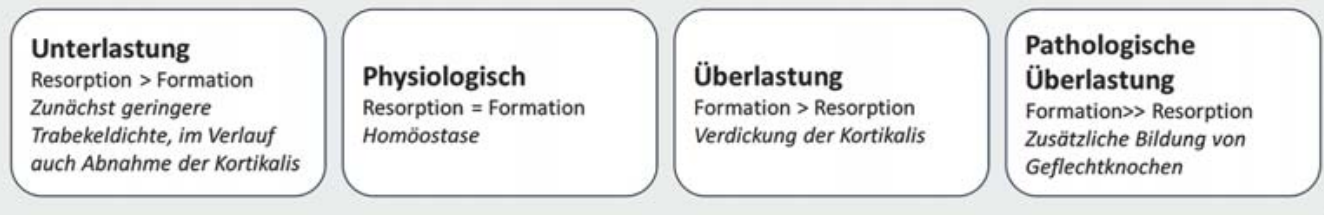

Belastung

- Abb. 2 Reaktion des Knochens auf mechanische Belastung. Die Stärke der Belastung, die notwendig ist, um ins nächste Fenster zu gelangen, ist abhängig von Größe, Form und Lokalisation innerhalb des Knochens und Verortung des Knochens im muskuloskelettalen System. 
erfolgen. Kompressionskräfte, die orthogonal (senkrecht) zur Frakturebene verlaufen, wirken sich im Gegensatz zu Scherkräften positiv auf die Bildung von Knochen aus [15]. Claes et al. konnten die mechanischen Bedingungen, unter denen die Differenzierung der Zellen stattfindet, näher einschränken und quantifizieren. Durch eine In-silico-Modellierung der Belastungen des heilenden Frakturkallus konnten für die einzelnen Abschnitte innerhalb des Kallus sowohl die mechanische Belastung als auch der hydrostatische Druck bestimmt werden [16]. Der Vergleich mit histologischen Schnitten im Großtiermodell hat gezeigt, welche Zelltypen sich unter diesen Bedingungen bilden; so fördern kleinere Belastungen und niedriger hydrostatischer Druck beispielsweise eine enchondrale Ossifikation. Komplexere In-silico- und In-vivo-Modelle von Checa et al. konnten die eingeschränkte Neovaskularisation bei erhöhter mechanischer Belastung belegen, sowie zeigen, dass sich die Zellen in Reaktion auf mechanische Reize der extrazellulären Matrix räumlich organisieren [17-19].

\section{Absolute und relative Stabilität}

Wenn eine Fraktur unter den Gesichtspunkten der absoluten Stabilität, also mit relativ hoher Steifigkeit und kaum Bewegungen im Frakturspalt fixiert werden kann, so kommt es zu einer primären Knochenheilung. Das heißt, dass Osteone von einem Fragment zum anderen den Spalt überbrücken, und so den Knochen durch Umbau wieder verbinden. Dies ist nur möglich, wenn eine ausreichende Kompression der Fraktur bei anatomischer Reposition erreicht wird.

Kommt es bei der Fixierung einer Fraktur zu einer relativen Stabilität mit gewissen Bewegungen im Frakturspalt, resultiert daraus eine indirekte Frakturheilung. Zu Beginn der Heilung wirken die Scher-, Biege- und Druckkräfte auf die Frakturkanten und führen zu Relativbewegungen der Fragmente, deren Ausmaß maßgeblich von der Steifgkeit der gewählten Osteosynthese abhängt. Im Frakturspalt bildet sich aus dem Hämatom zunächst vorwiegend Binde- und Knorpelgewebe, und nur periostal und endostal wird an den Knochenoberflächen, an denen keine Verformungen auftreten, neuer Knochen durch Apposition angelagert. Zwischen den sich bildenden Kallushälften wird nachgiebiges Bindegewebe eingelagert, und auf den einander zugewandten Oberflächen der Kallushälften kommt es zur Formation von Knorpelgewebe. Dieser noch weiche Frakturkallus verformt sich stark und wird vergleichbar dem Gelenkknorpel - in dieser Phase durch die passiven Flüssigkeitsverschiebungen auch ernährt, während die Blutgefäße anfangen einzuwachsen. [20]. Mit zunehmender Größe des Kallus nimmt die Beweglichkeit der Frakturkanten ab und es kommt letztlich periostal zum ersten Brückenschlag zwischen proximalem und distalem Fragment. Die Frakturzone selber wird zunehmend geringeren Scher-, Biege und Druckbewegun- gen ausgesetzt. Bei großen Torsions- und Biegebelastungen kommt es zu einem sehr ausladenden Kallus, der die Scherbewegungen deutlich reduzieren kann. Durch solche Gewebeformationen werden mechanische Bedingungen geschaffen, unter denen eine enchondrale Ossifikation, also eine Knochenbildung über die Zwischenstufe Knorpel (der dann mineralisiert), erfolgen kann. Parallel zur knöchernen Überbrückung erfolgt sehr zügig auch der Umbau des Kortex und des Knochens hin zu einem neu ausgerichteten, lamellären Knochengerüst, dass letztlich nach Monaten auch wieder die initialen mechanischen Eigenschaften des intakten Gewebes aufweist.

Um die Bedeutung der Mechanik für die Knochenheilung besser verstehen und auch bestimmen zu können ab wann die Osteosynthesesteifigkeit essenziell für die Heilung ist, wurde exemplarisch in einem Schaf die Steifigkeit der Osteosynthese zur Beschleunigung der Frakturheilung variiert: in einer In-vivo-Studie wurde der Heilungsverlauf der ersten 9 Wochen an einer $3 \mathrm{~mm}$ transversen Osteotomie der Tibia mit Osteosynthesen mit unterschiedlicher Steifigkeiten über die Zeit untersucht. Es konnte gezeigt werden, dass die nahezu intakte mechanische Kompetenz des Knochens nach 9 Wochen durch eine Osteosynthese mit einer axialen Steifigkeit von ca. $2200 \mathrm{~N} / \mathrm{mm}(1200-2700 \mathrm{~N} / \mathrm{mm})$ und einer Schersteifigkeit von ca. $430 \mathrm{~N} / \mathrm{mm}(120-470 \mathrm{~N} / \mathrm{mm})$ erreicht werden kann [21]. Geringere oder höhere axiale Steifigkeiten und geringere Schersteifigkeiten führten zu deutlich reduzierter mechanischer Kompetenz 9 Wochen postoperativ. Schafsknochen sind in der Regel etwas fester, aber auch spröder als humane Knochen. Dennoch können Belastbarkeit, Größe, Form und Beanspruchung in vivo mit denjenigen bei jungen Patienten verglichen werden. Damit ist die Untersuchung eine sehr positive Betrachtung des Einflusses der Osteosynthesesteifigkeit auf die Knochenheilung; bei älteren Patienten mit Begleiterkrankungen würde der Unterschied zwischen einer mechanisch „optimierten “ und einer weniger optimierten Heilung deutlich ausgeprägter ausfallen. Ideal für eine sekundäre Frakturheilung in einem 3-mm-Defektmodell ist eine relativ hohe Schersteifigkeit bei angepasster, moderater axialer Steifigkeit. Dies konnte auch in FE-Analysen des humanen Femurs eindrücklich nachgewiesen werden [22].

\section{Die Stabilität und die Osteosynthese}

Die Indikationsstellung zur operativen Therapie und Wahl des Osteosyntheseverfahrens ist abhängig von der Lokalisation und Morphologie der Fraktur sowie patientenspezifischen Faktoren (z.B. Alter, Nebenerkrankungen, Begleitverletzungen, etc.) Die verschiedenen Osteosyntheseformen bringen biomechanische Eigenschaften mit (absolute vs. relative Stabilität, dynamische vs. statische Kompression), die anwendungsabhängig unterschiedliche Konfigurationen zulassen. Plattenosteosynthesen erlauben beispielsweise in Abhängigkeit von Implantatlage, 

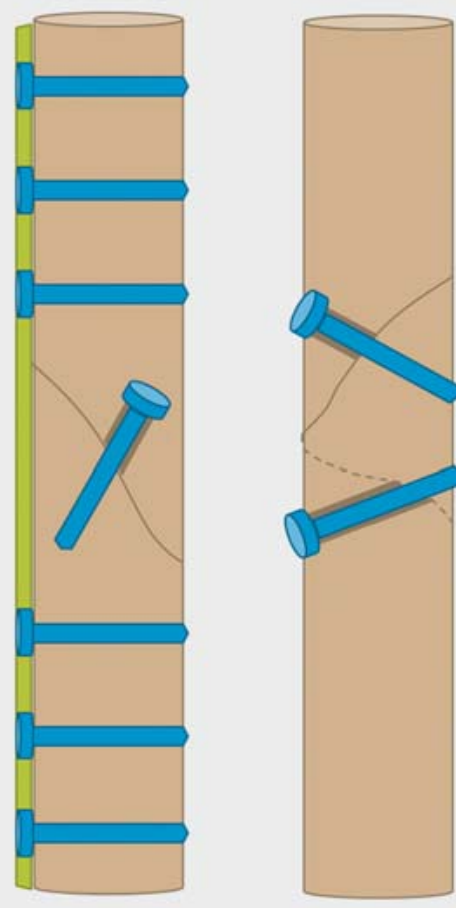

- Abb. 3 Zugschraubenosteosynthese in Kombination mit einer Neutralisationsplatte (links) und reine Zugschraubenosteosynthese (rechts).

Winkelstabilität und Schraubenplatzierungen eine Vielzahl an biomechanischen Konfigurationen, die sich leider nicht unmittelbar aus dem einzelnen Implantat, sondern erst durch dessen Konfiguration ergeben.

\section{Schraubenosteosynthesen}

Schraubenosteosynthesen können sowohl isoliert als auch ergänzend zu einer Plattenosteosynthese verwendet werden ( $\triangleright$ Abb. 3). Zugschraubenosteosynthesen werden meist genutzt, um Kompression im Frakturspalt zur primären Frakturheilung zu erzielen (absolute Stabilität). Häufige Anwendungsgebiete sind hier zum Beispiel Spiralfrakturen der kleinen Röhrenknochen, Skaphoidfrakturen (Herbert-Schraube) oder in Kombination mit einer Neutralisationsplatte bei Frakturen der distalen Fibula.

Bei der Kombination von Schrauben und Plattenosteosynthesen gilt es zu beachten, dass eine Zugschraube über dem Frakturspalt bei Verwendung einer winkelstabilen Platte eine erhebliche Erhöhung der Steifigkeit zur Folge hat [23]. Diese kann gegebenenfalls aufgrund einer unzureichenden mechanischen Stimulation an den Frakturkanten bei verbliebenem Frakturspalt zu einer deutlichen Verzögerung der Frakturheilung führen. Durch hohe Steifigkeit kann der mechanische Stimulus zu klein sein und Hämatom und lokale Zellen erhalten nicht die nötigen mechanischen Reize für eine schnelle Regeneration. Bei zu großer Steifigkeit kann es hilfreich sein, sekundär die Zugschraube wieder zu entfernen (Dynamisierung), um eine Frakturheilung anzuregen [24].

\section{Drahtosteosynthesen}

Reine Kirschner-Draht-Osteosynthesen erreichen nur eine relative Stabilität und können keine Kompression des Frakturspaltes erreichen. Insbesondere ist die Torsionsstabilität vermindert, was wiederum zu einer verlangsamten Heilung im Vergleich zu einer rigideren Stabilisierung führen kann. Oftmals resultiert die Notwendigkeit einer solchen Technik aus der Weichteilsituation oder dem Wunsch, Invasivität zu vermeiden. Dies vor allem bei der Stabilisierung von kleinen Knochen oder zur Schonung der Epiphysenfugen bei pädiatrischen Frakturen.

Eine besondere Form der Drahtosteosynthese stellt die sog. Zuggurtungsosteosynthese dar. Sie wirkt einem Muskelzug entgegen, der an 2 Fragmenten wirkt und den Frakturspalt asymmetrisch unter Scherung und Biegung belastet. Um eine Kompression der Frakturzone zu erreichen, ist es notwendig, auf der konvexen Seite Zugkräfte aufzunehmen, wobei auf der konkaven Seite die Druckkräfte noch erhöht werden. Die Zuggurtung wird als Schlinge am proximalen und distalen Frakturfragment quer fixiert und hat ihren Längsverlauf über der konvexen Fläche; so kann sie die Zugkräfte der konkaven Seite in Druckkräfte auf der gegenüberliegenden Seite umwandeln ( $\triangleright$ Abb. 4). Die Zuggurtung findet beispielsweise bei Patella- und Olekranonfrakturen Anwendung und stellt, richtig ausgeführt, eine Osteosynthese mit hoher Stabilität bei dynamischer Kompression dar. Wesentlich ist zur Sicherstellung der Stabilität eine adäquate Reposition und Vorspannung des Drahtes.

\section{Osteosynthesen mittels intramedullären Nägeln}

Marknägel sind zumeist lokalisationsspezifisch anatomisch vorgeformt und können in aufgebohrter oder unaufgebohrter Technik verwendet werden. Wird eine aufgebohrte Marknagelung durchgeführt, so lässt sich durch eine langstreckige Verklemmung eine erhöhte Stabilität erreichen. Die hohe Primärstabilität durch die Verklemmung im Markraum geht auf Kosten der endostalen Durchblutung. Die unaufgebohrte Marknagelung dagegen stabilisiert die Fragmente durch Verriegelungsschrauben oder Klingen, um eine Rotation oder Scherung der Fragmentenden zu verhindern, hierbei erfolgt weniger Schaden an der endostalen Durchblutung. Für die axiale Stabilität stehen, je nach Nagel, sowohl einfache Löcher als auch Längslöcher zur Verfügung. Letztere erlauben eine axiale Kompression der Fragmentenden. Entscheidend für die Steifigkeit der Frakturversorgung mit Marknägeln ist die Verblockung durch die Verriegelungsschrauben und der Durchmesser des Nagels. Der Nageldurchmessser sollte so groß wie möglich gewählt werden, um Scherung und Torsion der Fragmente zu mini- 


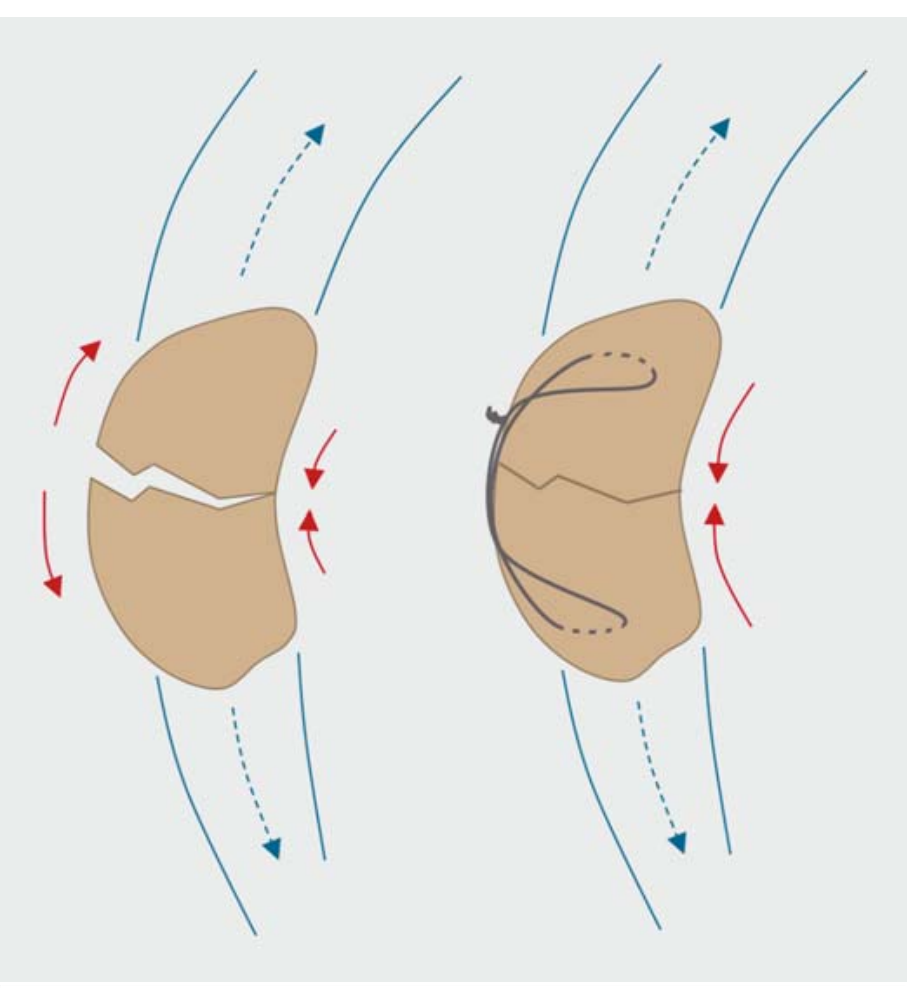

- Abb. 4 Zuggurtung am Beispiel einer Patella. Die Zugkräfte der konvexen Seite werden dank der Schlinge in Druckkräfte auf der konkaven Seite umgeleitet.

mieren. Oftmals ist der Isthmus der limitierende Faktor. So kann es gerade bei der unaufgebohrten Marknagelung zu erheblichen Fragmentbewegungen und damit zur Verzögerung der Heilung kommen.

\section{Plattenosteosynthesen}

Plattenosteosynthesen erreichen je nach Art der Osteosynthese durch unterschiedliche Mechanismen die Stabilisierung der Knochenfragmente. Eine Kompressionsplatte erzielt Stabilität über einen Zug, der über die Platte von einem Fragmentende auf das andere zu einer Kompression am Frakturspalt umgelenkt wird. Dabei wirkt eine Kompression zwischen Platte und Knochen, um letztlich auch den Frakturspalt stabilisieren zu können. Es wird diskutiert, ob durch die Kompression der Platte auf den Knochen die Durchblutung des Knochens und des Periosts vermindert wird. In jedem Fall erfolgt die Hauptbelastung aber vom Knochen über die Schrauben und Platte erneut in den Knochen, wobei die Spitzenlasten durch den Kompressionscharakter der Schrauben am Kontakt SchraubeKnochen auftritt. Auch hier erfolgen die größten knöchernen Anpassungen im weiteren Heilungsverlauf.

Im Gegensatz dazu versucht eine winkelstabile Platte, keinen Druck zwischen Platte und Knochen aufzubringen, und auch die Frakturenden werden nicht direkt unter Kompression gebracht. Vielmehr erfolgt die Lastüber- nahme über das verriegelte Platten-Schrauben-Konstrukt, wobei die Hauptlast in das Implantat verlagert wird. Daher sind auch die Schrauben-Knochen-Kontakte im Vergleich zur Kompressionsplatte deutlich entlastet; diese Stabilisierungen eignen sich besonders auch im schwachen knöchernen Lager (z.B. osteoporotischer Knochen). Auch ist die winkelstabile Platte als frakturüberbrückender Kraftträger bei Frakturen mit Trümmerzone gut einsetzbar, wenn die Schwinglängen der inneren Schrauben angemessen gewählt werden.

\section{Stabilität und interfragmentäre Bewegung}

Die interfragmentäre Bewegung bezeichnet die Maximalbewegung zwischen den Frakturfragmenten. Sie ist abhängig von 4 wesentlichen Faktoren: der Steifigkeit der verwendeten Osteosynthese, den Muskel- und Gelenkkräften, der Frakturgeometrie (auch knöcherne Abstützung unter Last) sowie der Steifigkeit des Gewebes im Frakturspalt. Die interfragmentäre Bewegung variiert über einen Frakturspalt, sodass die Heilung von unterschiedlichen Positionen aus beginnen kann. Da sich die Gesamtbewegung auf die Fragmente verteilt, haben Frakturen mit mehreren Fragmenten (Trümmerfrakturen) weniger Bewegung pro Flächeneinheit zwischen den einzelnen Fragmenten [14,21]. Größere interfragmentäre Bewegungen führen bei kleinen Frakturspalten zu vermehrter Kallusbildung. Jedoch kann bei größeren Frakturspalten eine zu große interfragmentäre Bewegung (besonders Scherung, also Bewegung parallel zur Frakturebene) zur Pseudarthrosenbildung beitragen [25]. Größere Frakturspalten führen zu vermehrter Bildung von Binde- und Knorpelgewebe und geringerer Vaskularisation trotz prozentual gleichbleibender Verformung (Normalisierung der Bewegung zur Spaltgröße) [26]. Eine Vielzahl an Faktoren beeinflusst die Steifigkeit der Osteosynthese und damit auch die interfragmentäre Bewegung.

Der Abstand der beiden frakturnahen Schrauben (erste Schraube proximal und distal der Fraktur) zueinander bestimmt die Schwingstrecke der Platte in einer winkelstabilen Osteosynthese ( $\bullet$ Abb.5). Eine größere Plattenschwingstrecke führt über eine Reduktion der Steifigkeit zu größerer interfragmentärer Bewegung. Steht die Platte in direktem Kontakt zum Knochen, wird die Steifigkeit nicht mehr nur durch den inneren Schraubenabstand beeinflusst, da die Schwingstrecke im Extremfall durch eine direkte Kraftübertragung vom Knochen auf die Platte bis auf die Größe des Frakturspaltes reduziert wird [27]. Bei längerer Schwingstrecke nehmen die Scherkräfte anteilig schneller zu als die axialen Kompressionskräfte. Die Schraubenpositionierung proximal und distal der frakturnähesten Schrauben macht, bei Verwendung von mindestens je 3 Schrauben proximal und distal der Fraktur, 
keinen signifikanten Unterschied bei der Gesamtsteifigkeit aus, jedoch verringern freie Löcher neben den frakturnahen Schrauben die Beanspruchung der Implantate, da die Platte sich mit weniger Einschränkung biegen kann [22].

Das Implantatmaterial hat ebenfalls Einfluss auf die Bewegung im Frakturspalt. Titan ist um ca. $40 \%$ elastischer als Stahl (dieser Wert kann abhängig der verwendeten Legierung etwas variieren). Bei einer Frakturversorgung mit absoluter Stabilität bzw. einer Frakturmorphologie, die noch eine kortikale Abstützung erlaubt, spielt das verwendete Implantatmaterial nur eine nachrangige Rolle. Je größer der Frakturspalt ohne kortikale Abstützung bzw. die Schwingstrecke der Platte, desto größer auch der Einfluss des verwendeten Materials auf die interfragmentäre Bewegung [28].

Die Verformung im Frakturspalt ist bei Versorgung mit einer Plattenosteosynthese bei Belastung asymmetrisch. Der größte Anteil der Verformung findet gegenüber der Platte statt ( $>$ Abb. 5). Unmittelbar unter der Platte findet kaum Bewegung statt. Ist der Abstand zwischen Osteosynthese und Belastungsachse größer, so ist eine stärkere Verformung bei gleicher Belastung möglich [29]. Dies kann durch erhöhte Materialbeanspruchung ein vorzeitiges Implantatversagen zur Folge haben [30].

Eine gleichmäßigere Verformung im Frakturspalt bei winkelstabilen Implantaten ist durch Verwendung von dynamischen winkelstabilen Schrauben oder sogenannten Active locking Plates zu erreichen [31,32]. Bislang hat sich jedoch keine dynamische Schraubenkonzeption durchsetzen können. Eine Weiterentwicklung solcher dynamischen Fixierungsansätze erscheint sehr sinnvoll und ist Gegenstand aktueller Forschung.

Da die gesamte Last in einer Fraktur ohne kortikale Abstützung bei Versorgung mit einer winkelstabilen Osteosynthese über diese umgeleitet wird, gilt bei Frakturen mit einseitiger kortikaler Trümmerzone zu beachten, dass diese Osteosynthesen bei vorhandener Abstützung auf der Gegenkortikalis (einfache, anatomisch reponierte Fraktur) eine sehr hohe Steifigkeit erzeugen können. Bei zu hoher Stabilität durch asymmetrischen Kontakt kann es mangels mechanischer Stimulation zu einer verzögerten Heilung kommen. Analoges gilt für Open-Wedge-Osteotomien [33].

\section{Stabilität und mögliche Komplikationen}

Eine inadäquat fixierte Fraktur kann zur Implantatlockerung oder dem Implantatbruch führen, was zumeist einer Revisionsoperation bedarf. Eine schwerwiegende Komplikation von nicht ausreichender oder zu großer Konstrukt-

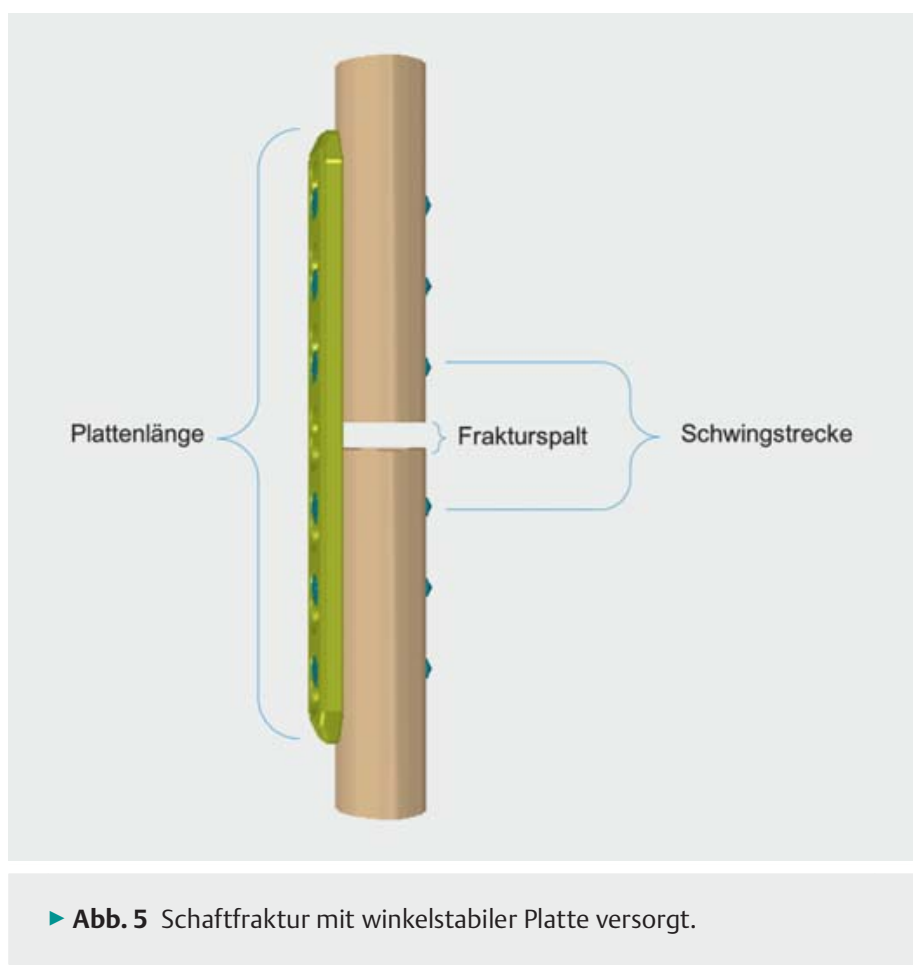

steifigkeit ist die Pseudarthrose. Insbesondere bei Frakturen mit großer Trümmerzone muss sichergestellt werden, dass die Osteosynthese nicht zu steif ist (führt über fehlende mechanische zu einer verzögerten Knochenheilung) oder zu flexibel (führt über zu hohe Scherbewegungen zur hypertrophen Pseudarthrose). Grundsätzlich gilt, dass in solchen Fällen die Versorgung eine axiale Kompression (Bewegung senkrecht zur Frakturebene) erlaubt, aber wesentliche Scherbewegungen und Torsionsbewegungen (Bewegung in der Frakturebene) vermieden werden sollten [34]. Derzeit scheint es, als ob zu steife Osteosynthesen (insbesondere in der unteren Extremität) häufiger zu gravierenden Komplikationen führen als zu flexible Osteosynthesen [35, 36].

In einigen Fällen ist die Therapie einer Pseudarthrose durch eine Re-Osteosynthese mit gegebenenfalls Anfrischung der Frakturenden möglich. Lässt sich hierdurch keine knöcherne Konsolidierung erreichen, so verbleibt meist ein größerer Defekt, welcher in Schwerpunktzentren mit aufwendigen operativen Eingriffen zu therapieren und mit langer Arbeitsunfähigkeit und hohen Kosten verbunden ist [37].

Der Chirurg kann bei seiner primären Versorgung die wesentlichen Bedingungen für eine schnelle Heilung beeinflussen und auch optimieren.

- Um eine einerseits stabile, aber andererseits auch hinreichend stimulierende Heilung zu ermöglichen, kann zum Beispiel die Plattenschwingstrecke als wesentlicher Einflussfaktor der Osteosynthesesteifigkeit bei winkelstabilen Verfahren so moduliert werden, dass 
Scherung in der Frakturebene minimiert und axiale Kompression optimiert wird.

- Mangelhafte Reposition gilt neben Rauchen als wesentlicher Einflussfaktor für eine verzögerte Knochenheilung [25]. Daher sollte auf eine möglichst gute Reposition geachtet werden.

- In großen Frakturspalten oder Trümmerzonen gilt zu beachten, dass mit steigender Schwingstrecke die Scherbewegung, welche die Heilung erheblich beeinträchtigt, überproportional ansteigt.

- Letztlich ist die Basis jeder Heilung das kompetente Frakturhämatom. Dieses soweit möglich in der Fraktur zu belassen und damit den Startpunkt der Heilung neben dem Schutz der Weichteile - zu respektieren, ist Grundlage jeder schnellen Heilung.

Mit diesen Punkten sind die Aspekte der Knochenheilung angerissen, die der Chirurg bei seiner primären Versorgung unmittelbar beeinflussen und damit auch optimieren kann. Mit zunehmendem Alter der Patienten und Zunahme der Nebenerkrankungen kommen andere Parameter hinzu, die auch bei optimaler Mechanik die Heilung wesentlich verlangsamen oder behindern können. Wesentlich ist sicher der immunologische Status des Patienten, aber durch die Beachtung einer hinreichenden axialen Kompression bei gleichzeitiger Minimierung der Scherung der Fragmentenden ist eine gute Basis für den biologischen Prozess der Knochenheilung gelegt.

\section{Fazit}

Die intrinsische regenerative Fähigkeit von Knochen ermöglicht zumeist die vollständige Wiedererlangung seiner Struktur und Funktion, ohne dass eine Narbe verbleibt. In Abhängigkeit von Frakturlokalisation, -morphologie und Patientenvoraussetzungen sollten Osteosynthesen so gewählt werden, dass dieses intrinsische Heilungspotenzial suffizient wirksam sein kann und nicht durch mechanische Instabilität oder zu rigide Fixation behindert wird. Die Abschätzung der notwendigen mechanischen Stabilität, um eine Knochenheilung zu unterstützen, erfordert neben fundierten chirurgischen Fähigkeiten ein ebenso fundiertes Verständnis der verfügbaren Osteosyntheseprinzipien, Fixationsmöglichkeiten sowie deren Einflussfaktoren.

\section{Interessenkonflikt}

Die Autoren geben an, dass kein Interessenkonflikt besteht.
Autorinnen/Autoren

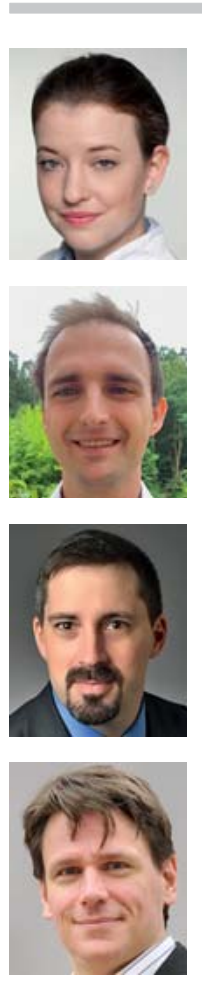

\section{Gabriele Rußow}

Assistenzärztin, Centrum für Muskuloskeletale Chirurgie, Charité - Universitätsmedizin Berlin

\section{Mark Heyland}

Dipl.-Ing., Wissenschaftlicher Mitarbeiter, Julius Wolff Institut für Biomechanik und Muskuloskeletale Regeneration, Charité - Universitätsmedizin Berlin

\section{Sven Märdian}

PD Dr. med., PhD, Kommissarischer Geschäftsführender Direktor, Centrum für Muskuloskeletale Chirurgie, Charité - Universitätsmedizin Berlin

\section{Georg N. Duda}

Univ.-Prof. Dr.-Ing., Institutsdirektor, Julius Wolff Institut für Biomechanik und Muskuloskeletale Regeneration, Charité - Universitätsmedizin Berlin

\section{Korrespondenzadresse}

Univ.-Prof. Dr.-Ing. Georg N. Duda

Centrum für Muskuloskeletale Chirurgie

Charité - Universitätsmedizin Berlin

Augustenburger Platz 1

13353 Berlin

Literatur

[1] Willie BM, Petersen A, Schmidt-Bleek K et al. Designing biomimetic scaffolds for bone regeneration: why aim for a copy of mature tissue properties if nature uses a different approach? Soft Matter 2010; 6: 4976-4987. doi:10.1039/c0sm00262c

[2] Hernigou P. Plaster of Paris: the orthopaedic surgeon heritage. Int Orthop 2016; 40: 1767-1779. doi:10.1007/s00264-0163179-2

[3] Mathijsen A. New method for application of plaster-of-Paris bandage. 1852. Clin Orthop Relat Res 2007; 458: 59-62. doi:10.1097/BLO.0b013e31803def0c

[4] Knothe U, Knothe Tate ML, Perren SM. 300 Years of Intramedullary Fixation - from Aztec Practice to Standard Treatment Modality. Eur J Trauma 2000; 5: 217-225

[5] Augat P, von Rüden C. Evolution of fracture treatment with bone plates. Injury 2018; 49: S2-S7. doi:10.1016/s00201383(18)30294-8

[6] Danis R. Treatment of fractures. Lancet 1947; 2: 520

[7] Giannoudis PV, Einhorn TA, Marsh D. Fracture healing: The diamond concept. Injury 2007; 38: S3-S6. doi:10.1016/s00201383(08)70003-2

[8] Giannoudis PV, Einhorn TA, Schmidmaier G et al. The diamond concept - open questions. Injury 2008; 39: S5-S8. doi:10.1016/s0020-1383(08)70010-x 
[9] Thompson WR, Rubin CT, Rubin J. Mechanical regulation of signaling pathways in bone. Gene 2012; 503: 179-193. doi:10.1016/j.gene.2012.04.076

[10] Epker BN, Frost HM. Periosteal Appositional Bone Growth from Age Two to Age Seventy in Man. Anat Rec 1966; 154: 573-578

[11] Frost HM. Bone "Mass" and the "Mechanostat": A Proposal. Anat Rec 1987; 219: 1-9

[12] Duncan RL, Turner $\mathrm{CH}$. Mechanotransduction and the Functional Response of Bone to Mechanical Strain. Calcif Tissue Int 1995; 57: 344-358

[13] Hadid A, Epstein Y, Shabshin N et al. Biomechanical Model for Stress Fracture-related Factors in Athletes and Soldiers. Med Sci Sports Exerc 2018; 50: 1827-1836. doi:10.1249/ mss. 0000000000001628

[14] Glatt V, Evans CH, Tetsworth K. A Concert between Biology and Biomechanics: The Influence of the Mechanical Environment on Bone Healing. Front Physiol 2016; 7: 678. doi:10.3389/fphys.2016.00678

[15] Kenwright J, Goodship AE. Controlled Mechanical Stimulation in the Treatment of Tibial Fractures. Clin Orthop Relat Res 1989; 241: 36-47

[16] Claes L, Haegele CA. Magnitudes of local stress and strain along bony surfaces predict the course and type of fracture healing. J Biomech 1999; 32: 255-266

[17] Checa S, Prendergast PJ. A mechanobiological model for tissue differentiation that includes angiogenesis: a lattice-based modeling approach. Ann Biomed Eng 2009; 37: 129-145. doi:10.1007/s10439-008-9594-9

[18] Checa S, Prendergast PJ. Effect of cell seeding and mechanical loading on vascularization and tissue formation inside a scaffold: a mechano-biological model using a lattice approach to simulate cell activity. J Biomech 2010; 43: 961-968

[19] Checa S, Rausch MK, Petersen A et al. The emergence of extracellular matrix mechanics and cell traction forces as important regulators of cellular self-organization. Biomech Model Mechanobiol 2015; 14: 1-13. doi:10.1007/s10237-014-0581-9

[20] Epari DR, Lienau J, Schell $\mathrm{H}$ et al. Pressure, oxygen tension and temperature in the periosteal callus during bone healing-an in vivo study in sheep. Bone 2008; 43: 734-739. doi:10.1016/j. bone.2008.06.007

[21] Epari DR, Kassi JP, Schell $\mathrm{H}$ et al. Timely fracture-healing requires optimization of axial fixation stability. J Bone Joint Surg Am 2007; 89: 1575-1585. doi:10.2106/JBJS.F.00247

[22] Mardian S, Schaser KD, Duda GN et al. Working length of locking plates determines interfragmentary movement in distal femur fractures under physiological loading. Clin Biomech (Bristol, Avon) 2015; 30: 391-396. doi:10.1016/j.clinbiomech.2015.02.006

[23] Mardian S, Schmolz W, Schaser KD et al. Interfragmentary lag screw fixation in locking plate constructs increases stiffness in simple fracture patterns. Clin Biomech (Bristol, Avon) 2015; 30: 814-819. doi:10.1016/j.clinbiomech.2015.06.008

[24] Oh JK, Hwang JH, Lee SJ et al. Dynamization of locked plating on distal femur fracture. Arch Orthop Trauma Surg 2011; 131: 535-539. doi:10.1007/s00402-010-1202-4
[25] Claes L, Augat P, Suger G et al. Influence of Size and Stability of the Osteotomy Gap on the Success of Fracture Healing. J Orthop Res 1997; 15: 577-584

[26] Claes L, Eckert-Hubner K, Augat P. The fracture gap size influences the local vascularization and tissue differentiation in callus healing. Langenbecks Arch Surg 2003; 388: 316-322. doi:10.1007/s00423-003-0396-0

[27] MacLeod AR, Pankaj P. Pre-operative planning for fracture fixation using locking plates: device configuration and other considerations. Injury 2018; 49: S12-S18. doi:10.1016/s00201383(18)30296-1

[28] Heyland M, Duda GN, Mardian S et al. [Steel or titanium for osteosynthesis: A mechanobiological perspective]. Unfallchirurg 2017; 120: 103-109. doi:10.1007/s00113-016-0289-7

[29] MacLeod AR, Simpson AH, Pankaj P. Age-related optimization of screw placement for reduced loosening risk in locked plating. J Orthop Res 2016; 34: 1856-1864. doi:10.1002/ jor.23193

[30] Ahmad M, Nanda R, Bajwa AS et al. Biomechanical testing of the locking compression plate: when does the distance between bone and implant significantly reduce construct stability? Injury 2007; 38: 358-364. doi:10.1016/j.injury.2006.08.058

[31] Henschel ], Tsai S, Fitzpatrick DC et al. Comparison of 4 Methods for Dynamization of Locking Plates: Differences in the Amount and Type of Fracture Motion. J Orthop Trauma 2017; 31: 531-537. doi:10.1097/BOT.0000000000000879

[32] Heyland M, Duda GN, Haas NP et al. Semi-rigid screws provide an auxiliary option to plate working length to control interfragmentary movement in locking plate fixation at the distal femur. Injury 2015; 46 (Suppl.4): S24-S32. doi:10.1016/ s0020-1383(15)30015-2

[33] Roderer G, Gebhard F, Duerselen L et al. Delayed bone healing following high tibial osteotomy related to increased implant stiffness in locked plating. Injury 2014; 45: 1648-1652. doi:10.1016/j.injury.2014.04.018

[34] Augat P, Margevicius K, Wolf S et al. Local Tissue Properties in Bone Healing: Influence of Size and Stability of the Osteotomy Gap. J Orthop Res 1998; 16: 475-481

[35] Harvin WH, Oladeji LO, Della Rocca G] et al. Working length and proximal screw constructs in plate osteosynthesis of distal femur fractures. Injury 2017; 48: 2597-2601. doi:10.1016/j. injury.2017.08.064

[36] Strauss EJ, Schwarzkopf R, Kummer F et al. The Current Status of Locked Plating: The Good, the Bad, and the Ugly. J Orthop Trauma 2008; 22: 479-486

[37] Giannoudis PV, Gudipati S, Harwood P et al. Long bone nonunions treated with the diamond concept: a case series of 64 patients. Injury 2015; 46 (Suppl. 8): S48-S54. doi:10.1016/ s0020-1383(15)30055-3

Bibliografie

DOI https://doi.org/10.1055/a-0755-6202 Online-publiziert 17.01.2019 | OP-JOURNAL 2019; 35: 12-19 (c) Georg Thieme Verlag KG Stuttgart · New York ISSN 0178-1715 